SUPPORTING INFORMATION

\title{
NIR Fluorescent Imaging and Photodynamic Therapy with a Novel Theranostic Phospholipid Probe for Triple-Negative Breast Cancer Cells
}

Natalia I. Rubtsova ${ }^{1}$, Michael C. Hart' ${ }^{1}$, Alejandro D. Arroyo ${ }^{1,2}$, Sofya A. Osharovich ${ }^{1}$, Benjamin K. Liebov $^{1,3}$, Joann Miller ${ }^{4}$, Min Yuan ${ }^{4}$, Jeffrey M. Cochran ${ }^{5}$, Sanghoon Chong ${ }^{5}$, Arjun G. Yodh ${ }^{5}$, Theresa M. Busch $^{4}$, E. James Delikatny ${ }^{1}$, Nadia Anikeeva ${ }^{6}$, Anatoliy V. Popov*1

${ }^{1}$ Department of Radiology, Perelman School of Medicine, University of Pennsylvania, 3620 Hamilton Walk, Philadelphia, Pennsylvania 19104, United States

${ }^{4}$ Department of Radiation Oncology, Perelman School of Medicine, University of Pennsylvania, 3400 Civic Center Blvd, Bldg 421, Philadelphia, Pennsylvania 19104, United States

${ }^{5}$ Department of Physics and Astronomy, University of Pennsylvania, 3231 Walnut Street, Philadelphia, Pennsylvania 19104, United States

${ }^{6}$ Department of Microbiology and Immunology, Thomas Jefferson University, Philadelphia, Pennsylvania 19107, United States

\section{TABLE OF CONTENT}

Scheme S1. Synthesis of LysoPtdEtn-QSY and Pyro-PtdEtn-QSY.

Synthesis of LysoPtdEtn-QSY

Synthesis of Pyro-PtdEtn-QSY

Scheme S2. Synthesis of Pyro-PtdCho

Synthesis of Pyro-PtdCho

Figure S1. Nanoparticle size distribution, measured in a dynamic light scattering experiment, using DSPEPEG(1000) nanoparticles carrying no photosensitizer

Figure S2. Relative PC-PLC activity in breast cancer cell lines

Table S1. Fluorescence Intensity from various cell types measured by flow cytometry

Figure S3. Uptake of nanoparticles and quenched probe activation as measured by flow cytometry

Figure S4. D609 induce inhibition of Pyro-PtdEtn-QSY probe activation

Figure S5. Fluorescence Diffuse Optical Tomography results.

*Corresponding author, avpopov@pennmedicine.upenn.edu

${ }^{2}$ Current address: Memorial Sloan Kettering Cancer Center, 1275 York Avenue, New York, NY 10065

${ }^{3}$ Current address: Adesis, Inc, 27 McCullough Drive, New Castle, DE 19720, USA 


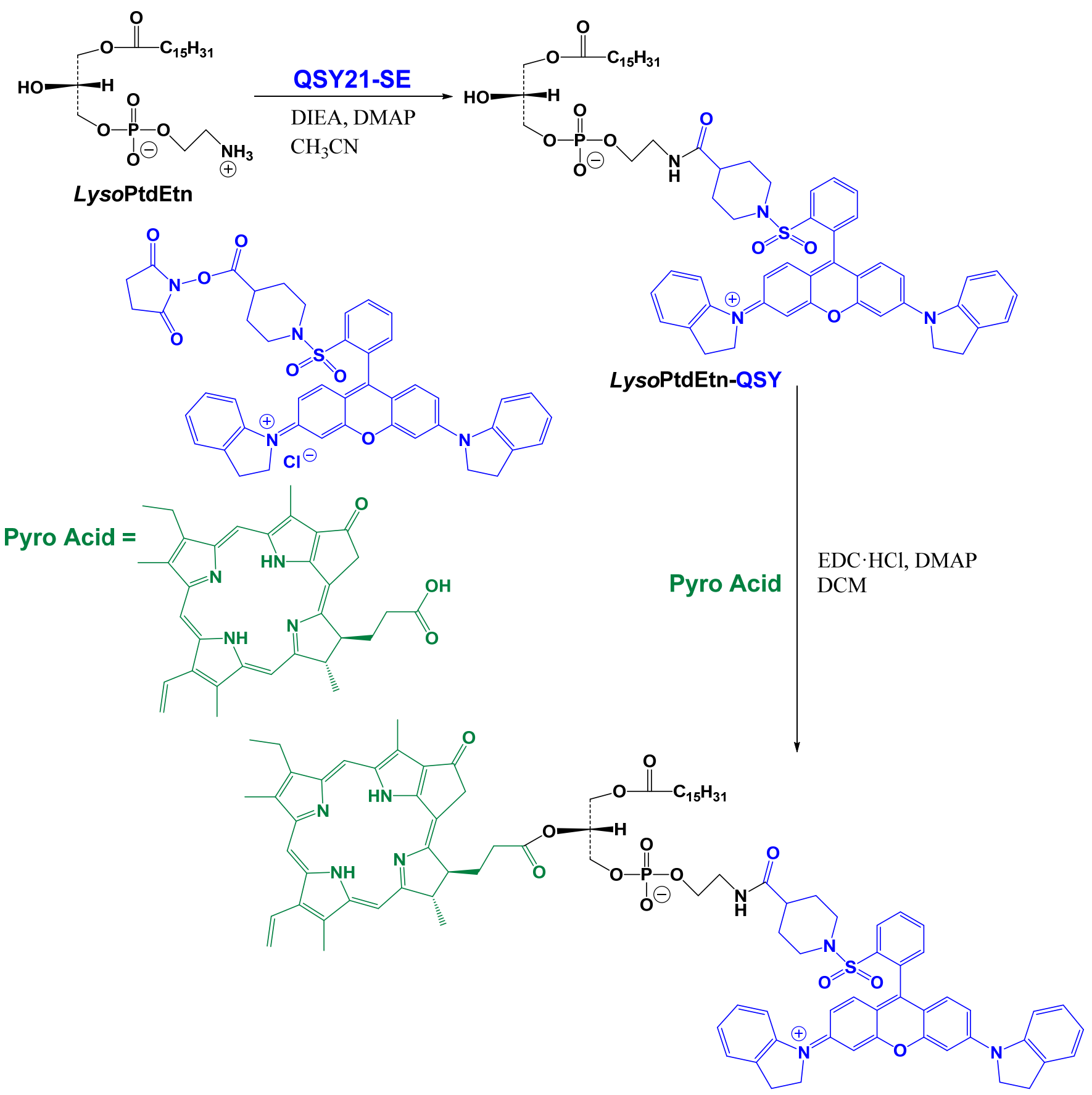

Pyro-PtdEtn-QSY

Scheme S1. Synthesis of LysoPtdEtn-QSY and Pyro-PtdEtn-QSY.

Synthesis of LysoPtdEtn-QSY. LysoPtdEtn (0.050 g, $0.110 \mathrm{mmol})$ and QSY-SE $(0.020 \mathrm{~g}, 0.0245 \mathrm{mmol})$ were added to a $100 \mathrm{~mL}$ round bottom flask with stir bar, followed by dry acetonitrile $(50 \mathrm{~mL})$, diisopropylethylamine $(40 \mu \mathrm{L}$, $0.230 \mathrm{mmol}$ ) and 4-(dimethylamino)pyridine (DMAP, $0.0087 \mathrm{~g}, 0.0712 \mathrm{mmol}$ ). The reaction flask was purged with argon and covered in aluminum foil. The blue reaction solution was stirred for 2 days. Reaction progress was tested via TLC on functionalized silica diol (Sorbtech cat \# 2933187) using a methanol:chloroform solution (5:95). The product was observed at $R_{f}=0.47$. The reaction solution was concentrated in vacuo until $\sim 3 \mathrm{~mL}$ remained. The 
reaction solution was loaded onto a silica diol (Sorbtech cat \# 62570-01) column. A gradient of methanol:chloroform eluent was used (0:100 to 20:80) to elute several blue fractions. The blue fractions were tested with TLC and based on these results the proper fractions were concentrated to a dark blue oil. The oil was left under vacuum overnight to reveal the product $(0.0239 \mathrm{~g}, 0.0214 \mathrm{mmol}, 87 \%$ yield). The product, LysoPtdEtnQSY, was confirmed by MALDI mass spectrometry and UV-Vis.

Synthesis of Pyro-PtdEtn-QSY. LysoPtdEtn-QSY (0.0190 g, $0.0170 \mathrm{mmol})$ was put into a 20 dram vial charged with a stir bar, followed by Pyro $(0.0118 \mathrm{~g}, 0.0542 \mathrm{mmol}), N$-(3-dimethylaminopropyl)- $N$ '-ethyl carbodiimide hydrochloride (EDC. $\mathrm{HCl}, 0.1848 \mathrm{~g}, 0.9640 \mathrm{mmol})$, and DMAP $(0.0225 \mathrm{~g}, 0.1841 \mathrm{mmol})$ and dry DCM ( $4 \mathrm{~mL})$. The reaction vial was purged with argon, covered in foil, and allowed to stir for $72 \mathrm{~h}$. The reaction was monitored by TLC on silica diol plates $\left(5: 95\right.$, methanol:chloroform) until an aquamarine spot appeared at $R_{f}=0.6$. The reaction solution was loaded onto a silica diol (Sorbtech cat \# 62570-01) column ( $0.5 \mathrm{~cm}$ ). Methanol:chloroform (1:99) was used to elute an aquamarine band. The aquamarine band was concentrated to an oil and placed under vacuum overnight to yield an aquamarine oil. The oil was dissolved in minimal chloroform and placed on Silica Diol preparative TLC plates. The plates were eluted with 10:90 methanol:chloroform. An aquamarine band was collected between $R_{f} S 0.70$ and 0.75 . The band scrapped from the plates, collected and stirred with 10:90 methanol:chloroform. After filtering off the sorbent the solution was concentrated and underwent a second preparative TLC to get rid of fluorescent impurities (Pyro acid). The product was isolated as an oil that was lyophilized overnight $(0.0194 \mathrm{~g}, 0.0119 \mathrm{mmol}, 70 \%$ yield). The overall yield of this two-step-synthesis is $61 \%$. The product was confirmed by MALDI mass spectrometry and UV-Vis.
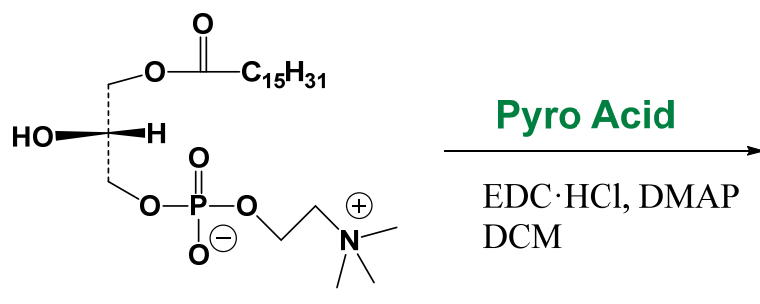

LysoPtdCho

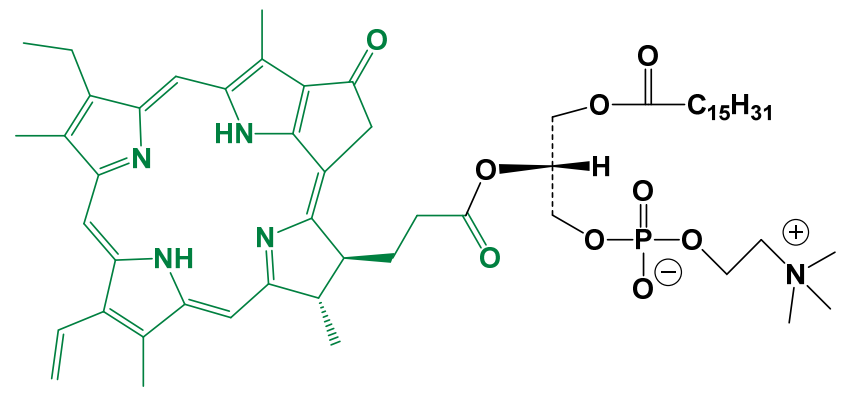

Pyro-PtdCho

Scheme S2. Synthesis of Pyro-PtdCho.

Synthesis of Pyro-PtdCho. LysoPtdCho (0.050 g, $0.101 \mathrm{mmol})$, pyropheophorbide $a$ acid (Pyro acid) $(0.050 \mathrm{~g}$, $0.094 \mathrm{mmol}), \mathrm{EDC} \cdot \mathrm{HCl}(0.186 \mathrm{~g}, 0.970 \mathrm{mmol}), 4$-(dimethylamino) pyridine (DMAP) $(0.114 \mathrm{~g}, 0.090 \mathrm{mmol})$ and DCM $(100 \mathrm{~mL})$ were combined in a $200 \mathrm{~mL}$ round bottom flask with stir bar. The reaction mixture was stirred at room temperature under argon in the dark for 48 hours. The solvent was evaporated and the residue was subjected twice to chromatography purification on Silica Diol preparative TLC plates. The plates were eluted with 10:90 methanol:chloroform. A wide dark green band was collected between $R_{f} S 0.2$ and 0.3. The compound contains an impurity, 1- pyropheophorbide $a$-2- palmitoyl-sn-glycero-3-phosphocholine, a product of 1,2-acyl shift (see refs 57, 59). The collected silica was stirred with 10:90 methanol:chloroform. After filtering off the sorbent the solvent was evaporated and Pyro-PtdCho was dried under high vacuum. The purity was confirmed by MALDI-TOF and UV-VIS. Yield $0.0247 \mathrm{~g}, 0,024 \mathrm{mmol}, 26 \%$ 


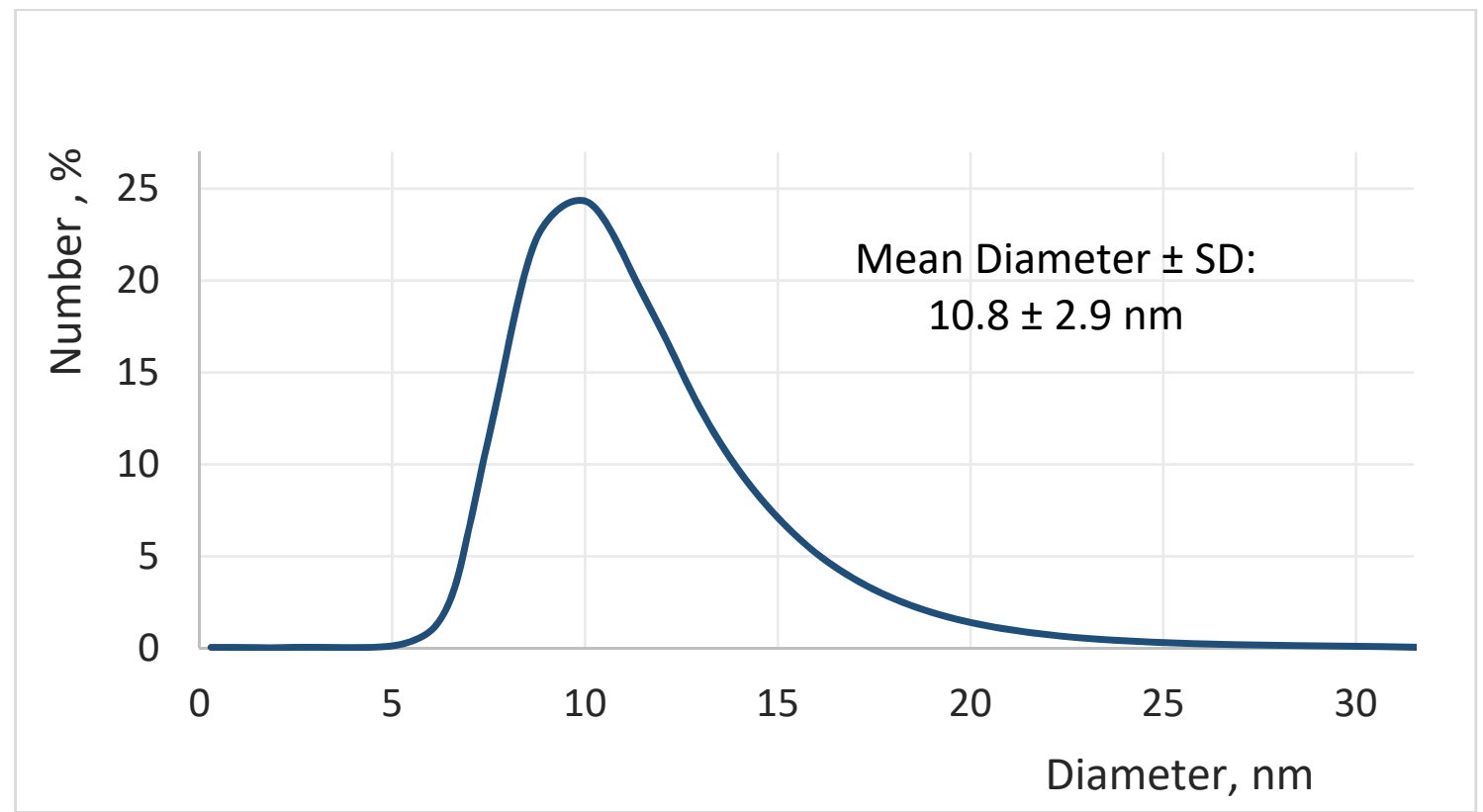

Figure S1. Nanoparticle size distribution, measured in a dynamic light scattering experiment, using DSPEPEG(1000) nanoparticles carrying no photosensitizer. 


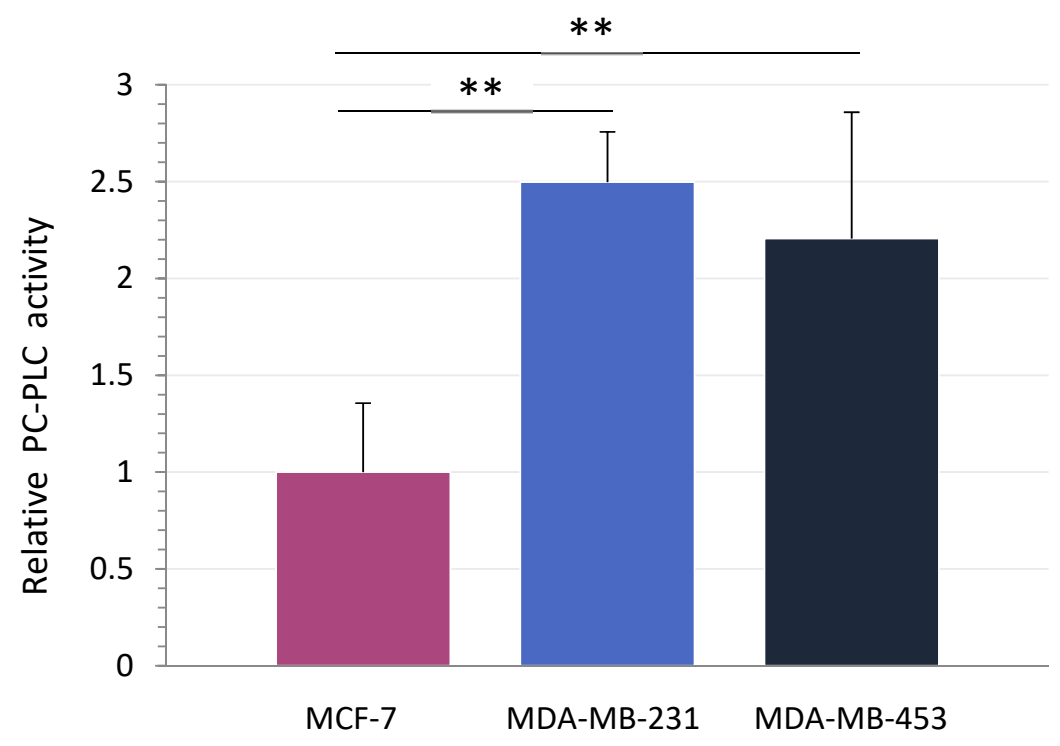

Figure S2. Relative PC-PLC activity in breast cancer cell lines. Activity was measured in total cellular lysates in triplicates using the Amplex Red Assay. Mean and SD are calculated, and the error bars are indicated for each mean value $(\mathrm{N}=3)$. There is a statistically significant difference in PC-PLC activity level between MCF7 cells and both MDA-MB-231 and MDA-MB-453 cell lines $(p<0.01)$ as determined by one-way ANOVA followed by Tukey post hoc test (GraphPad Prizm).

Table S1. Mean Fluorescence Intensity from various cell types measured by flow cytometry (a.u)

\begin{tabular}{lcccc}
\hline & MDA-MB-231 & MDA-MB-453 & SKBR3 & MCF7 \\
\hline 13.5\% Pyro-PtdEtn-QSY & $5130 \pm 230$ & $5298 \pm 201$ & $4587 \pm 238$ & $2266 \pm 173$ \\
13.5\% Pyro-PtdCho & $606 \pm 475$ & $544 \pm 181$ & $627 \pm 376$ & $260 \pm 207$ \\
\hline
\end{tabular}

Data were obtained following $6 \mathrm{~h}$ incubation of different breast cancer cell lines with $1 \mathrm{mM}$ of fluorescent probes at the given concentrations prepared in lipid nanoparticles. The means and standard deviations of three independent experiment are shown. 


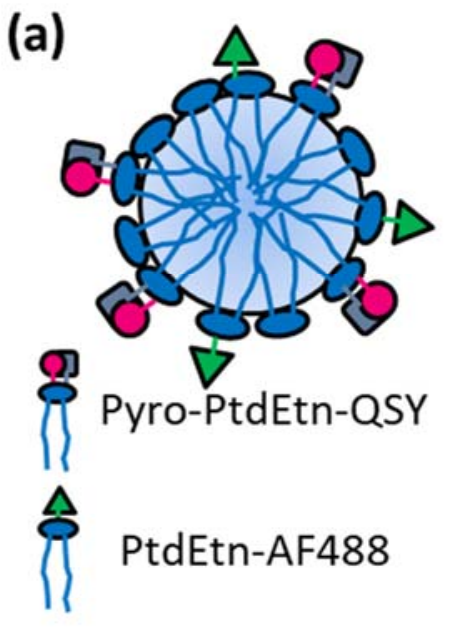

(a)

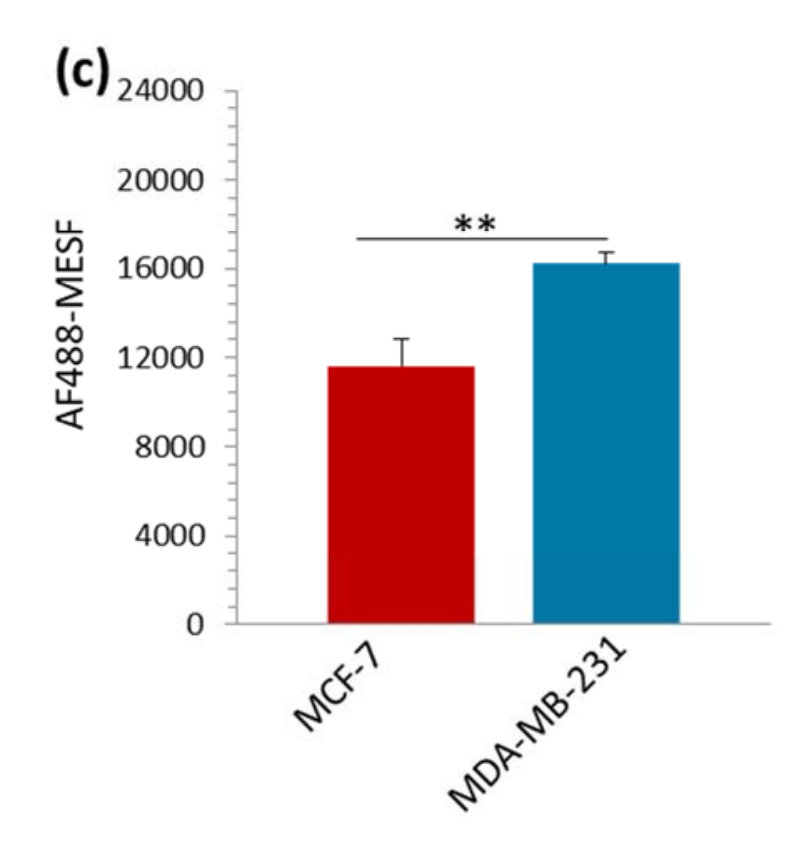

(b)

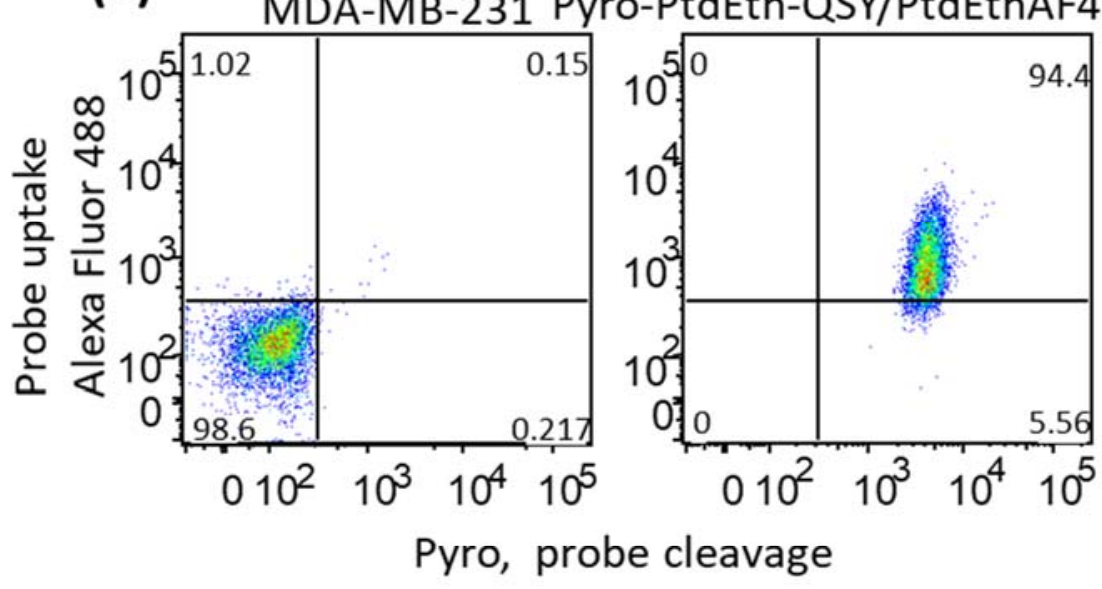

MDA-MB-231+

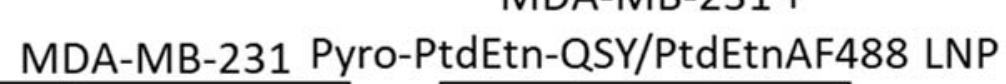

MDA-MB-231 Pyro-PtdEtn-QSY/PtdEtnAF488 LNP

Pyro, probe cleavage

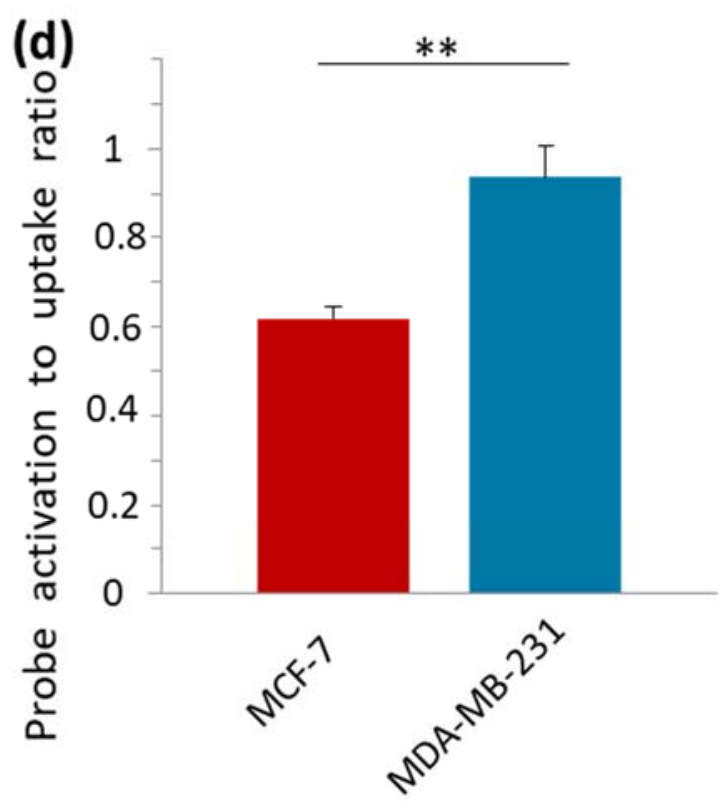

Figure S3. Uptake of nanoparticles and quenched probe activation as measured by flow cytometry. MCF-7 and MDA-MB-231 cells were treated with $1 \mu \mathrm{M}$ of mixed micelles containing both Pyro-PtdEtn-QSY and PtdEtnAF488 (a). AF488 and Pyro fluorescent intensities were measured by flow cytometry (right) relative to control cells with no probe added (left) (b). Median AF488 intensities of the nanoparticle-treated cells were converted to MESF using calibrating beads. Nanoparticles uptake is shown as average number of AF488 fluorophore per cell (c). Pyro-PtdEtn-QSY probe activation measured as mean of Pyro fluorescence intensity was normalized relative to the number of AF488 molecules (d). Each experimental point shows averages and standard deviations of three independent experiments. 


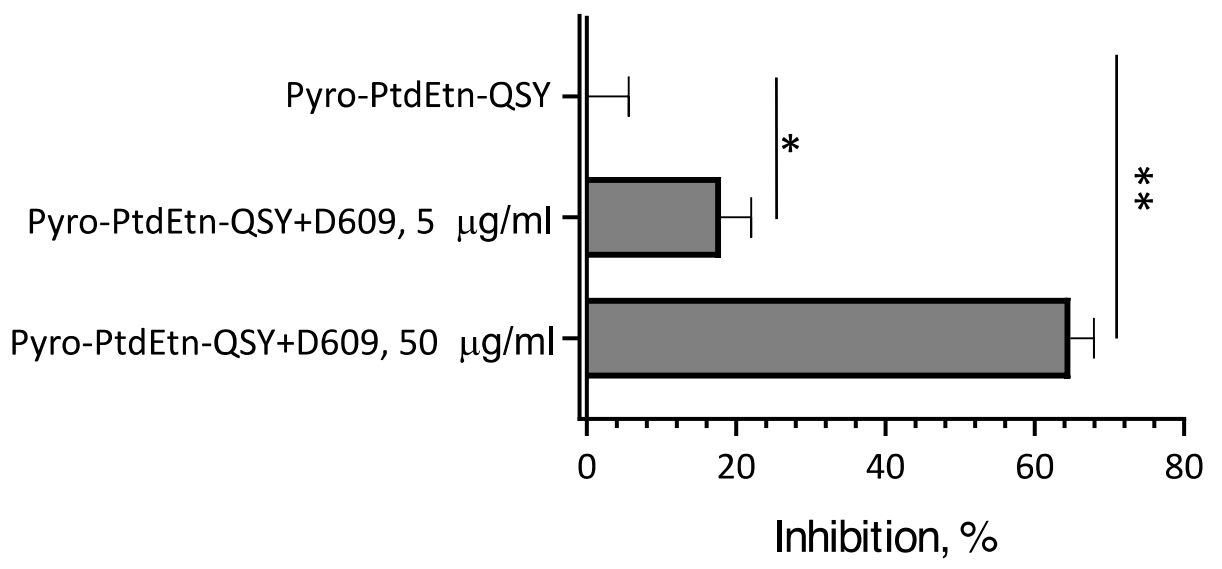

Figure S4. D609 inhibits Pyro-PtdEtn-QSY probe activation in MDA-MB-231 cells. MDA-MB-231 cells were exposed to the PC-PLC inhibitor at 5 or $50 \mu \mathrm{g} / \mathrm{mL}$ for 1 hour followed by incubation with $1 \mu \mathrm{M}$ of Pyro-PtdEtnQSY for one more hour. The probe activation was measured by flow cytometry, and inhibition was evaluated relative to untreated controls. Each bar represent mean \pm SD of three independent experiments. 
(a)

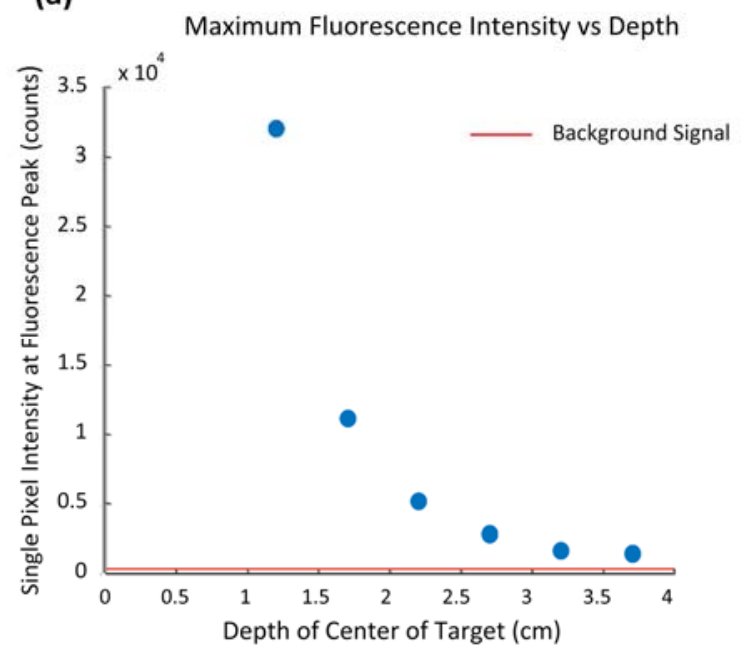

(b) Normalized Reconstructed Fluorophore Concentration

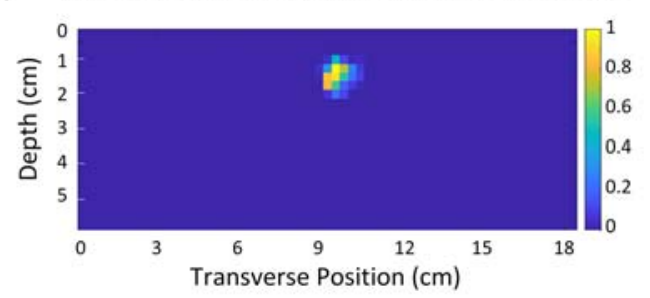

(c)

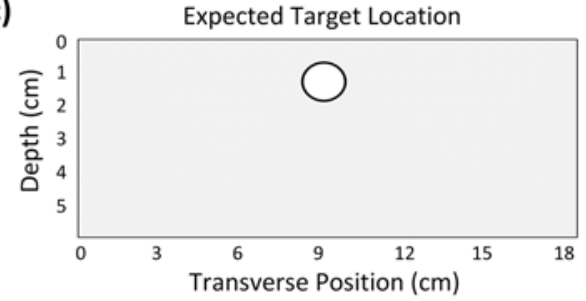

Figure S5. Fluorescence Diffuse Optical Tomography results. (a) Depth dependent Maximum Fluorescence Intensity. (b) Diffuse Optical Tomography reconstruction for the reflection geometry, when the target is centered at $1.2 \mathrm{~cm}$ depth (c) Expected location for the target. 